\title{
Discussion on the Effect of Tai Chi on Health Care of Middle-aged and Elderly Patients with Chronic Diseases
}

\author{
Wang Xudong ${ }^{1 \mathrm{a}}$, Zhang Yuqin $\mathrm{b}^{2 \mathrm{~b}^{*}}$ \\ ${ }^{1}$ Sports Department, Shaanxi University of Chinese Medicine, Xian yang, Shaanxi, China \\ ${ }^{2}$ Student, Shaanxi University of Chinese Medicine \\ Corresponding author. Email: $a^{*} 790086212 @ q q$. com $^{b^{*}} 2934874624 @ q q . c o m$
}

\begin{abstract}
this paper, by looking for references and other ways, through the analysis of the effect of Tai Chi on health care of middle-aged and elderly patients with chronic diseases, we know that Tai Chi has a certain effect on the daily health care and rehabilitation treatment of middle-aged and elderly patients with chronic diseases. not cite references in the abstract. Please do not place or cite tables and figures in the abstract either.
\end{abstract}

Keywords: Tai Chi, Patients with chronic diseases, health care

\section{INTRODUCTION}

Tai Chi is one of the three traditional Chinese martial arts, which integrates martial arts and health care, and is the essence of Chinese traditional culture. After the founding of the People's Republic of China, Tai Chi has been more and more accepted and practiced. Health, education, sports and other departments have listed Tai Chi as an important project to promote. Tai Chi has also attracted a lot of attention abroad, many countries have set up Tai Chi associations and other groups, attracting a large number of fans.

This template, modified in MS Word 2007 and saved as a "Word 97-2003 Document" for the PC, provides authors with most of the formatting specifications needed for preparing electronic versions of their papers. All standard paper components have been specified for three reasons: (1) ease of use when formatting individual papers, (2) automatic compliance to electronic requirements that facilitate the concurrent or later production of electronic products, and (3) conformity of style throughout conference proceedings.

\section{THE EFFECT OF TAI CHI ON THE PREVENTION OF CHRONIC DISEASES IN MIDDLE-AGED AND ELDERLY PEOPLE}

Due to the decline of physiological function and body immunity, the elderly's immunity to diseases is also reduced, and it is more likely to develop into chronic diseases. In today's society, due to poor diet, people suffering from chronic diseases are gradually becoming younger and younger. It is urgent to find a simple and effective way to prevent chronic diseases.

In the group of middle-aged and elderly patients with chronic diseases, even if they suffer from the same kind of chronic disease, due to individual differences between middle-aged and elderly people and some external factors, the clinical symptoms and signs of patients with chronic diseases are not typical or even greatly different. The vast majority of clinical patients with chronic diseases also have the characteristics of coexistence of multiple diseases, severe illness, rapid change, susceptible occur consciousness disorders, and many complications. And because the elderly people have low immunity and multiple organ function failure, the chance of complete recovery is very small. Therefore, the prevention of chronic diseases is very important.

As a health care sport with a long history in China, Tai Chi has its unique advantages in preventing chronic diseases in the middle and old age. People suffering from chronic diseases are mostly due to the decline of their daily diets and internal function of their body. Therefore, in addition to the healthy diet, the more important thing to prevent chronic diseases is to improve the body function. Tai Chi can consume fat, 
regulate body weight, promote digestion and absorption, enhance metabolism and enhance cardiovascular function. Long-term practice of Tai Chi can significantly improve the body's serum cortisol content, effectively improve the function of $\mathrm{T}$ and $\mathrm{B}$ lymphocytes, and improve the body immunity in the middle-aged and elderly. The practice of Tai Chi can also play a positive role in the mental health of middle-aged and elderly people.

Tai Chi can also enhance the physique of middle-aged and elderly people and improve the health qi inside human body. Tai Chi can harmonize the Yin and Yang of the human body and strengthen the circulation of Qi and blood, so as to achieve the effect that "the healthy Qi is stored in the body and evil do not save". For the middle-aged and old people who have already suffered from chronic diseases, long-term exercise can improve the sleep and mental state of patients with chronic diseases, make patients energetic, happy, and reduce the pain caused by the disease.

\section{THE EFFECT OF TAI CHI ON THE TREATMENT OF MIDDLE-AGED AND ELDERLY PATIENTS WITH CHRONIC DISEASES}

\subsection{The effect of Tai Chi on chronic respiratory diseases}

Common chronic respiratory diseases mainly include tuberculosis, chronic bronchitis, chronic obstructive emphysema, pulmonary heart disease, bronchial asthma, etc., which are mostly due to the pulmonary ventilation disorders and pulmonary ventilation disorders caused by the decay of lung function.

From the perspective of traditional Chinese medicine, the practice of Tai Chi should keep breathing naturally. Through thin, long, slow and even abdominal breathing method, it can effectively enhance the air capacity of the chest, increase the number of times of inhaling oxygen and exhaling carbon, ensure that the gas can be fully exchanged and improve the oxygen intake of each organ relatively. The practice of Tai Chi can effectively improve the lack of kidney function, to reduce the disease caused by asthma and so on. From the perspective of modern western medicine, Tai Chi emphasizes the regulation of central nervous system on breathing, and makes breathing dominated by consciousness. The main part of Tai Chi is abdominal breathing, which can effectively reduce the ventilation resistance during breathing and improve the alveolar ventilation volume, so as to improve the damage to the airway caused by a large amount of mucus and smooth muscle spasm caused by long-term lung inflammation in patients with chronic diseases. The long-term lung disease will reduce the activity of alveoli and the number of alveoli participating in ventilation significantly, then lead to the increase of capillary resistance and obvious vascular degeneration around alveoli, further lead to hypoxia and hypercapnia in patients. The requirement of Tai Chi "Qi sinks into the Dantian" and the strengthening of the functions of lung descending and all vessels converging in lung can effectively promote blood return, accelerate gas exchange and increase the blood flow circulation in the lung. So as to improve symptoms of dyspnea and ischemia and other symptoms caused by chronic lung diseases.

\subsection{Effects of Tai Chi on chronic digestive system diseases}

Chronic digestive system diseases, including all sorts of inflammation, functional gastrointestinal disease, autoimmune liver disease, and esophageal cancer, intestinal tuberculosis, constipation, fatty liver disease and primary liver cancer, hepatic encephalopathy, chronic pancreatitis, pancreatic cancer, gastrointestinal bleeding, etc., are mostly due to immoderate die, preference of spicy food, or some emotional problems.

Emotion is based on viscera function, and Tai Chi has a good effect on improving people's emotion. Tai Chi can enable patients to achieve a state of physical and mental peace by sinking Qi into Dantian and calming the heart, so as to reduce the influence of bad psychology on digestive system.,

The abdominal breathing used in Tai Chi can increase the diaphragm movement, generate a massage-like effect in the viscera, make the triple energizer Qi unobstructed, then promote gastrointestinal peristalsis, increase the secretion of gastric acid, accelerate gastrointestinal blood circulation, further effectively improve the appetite of patients, gastrointestinal dyspepsia, chronic gastroenteritis and other diseases. After practicing Tai Chi, patients will have a significant increase in appetite. By increasing the intake of nutrition, patients can get sufficient nutrition, plump muscles and strong and flexible limbs, which plays a good role in improving patients' own immunity and other adverse symptoms. In other words, Tai Chi can enhance the ability of patients with chronic digestive system diseases to absorb food and nutrition and speed up the healing of peptic ulcer. 


\subsection{The effect of Tai Chi on improving the function of the immune system}

The immune system is the body's most important defense against the attack of external viruses. For the middle-aged and elderly, due to the deterioration of immune function, their probability of suffering from disease is gradually increasing. From a theoretical point of view, "healthy qi s stored in the body, evil cannot be done", "if evil gather together, healthy Qi will be empty". The practice of Tai Chi can effectively improve the body's healthy qi, improve the quality of the human body, make the body and mind healthy, not easy to get sick. By comparing the changes of the main immune cells (such as T cell subsets, NKT cells, etc.) in the blood of middle-aged and elderly people before and after six months of Tai Chi exercise, as well as the changes of psychological indicators such as cognition and emotion of boxing practitioners, it was found that the number and proportion of immune cells in the body increased, and the immune function improved significantly. Practice has proved that long-term correct practice of Tai Chi can effectively improve human immunity, increase the number and activity of immune cells in circulating blood, so as to strengthen immunity to the outside world. Tai Chi is gentle, slow and habitual. Compared with the general sports, its intensity and amount of exercise are relatively small, so it is more suitable for middle-aged and elderly people.

Kidney is the innate foundation, which has an important impact on the immune system of the human body. The most obvious physiological change of middle-aged and elderly people is the attenuation of renal function, which leads to the decline of healthy qi deficiency and immune function. Tai Chi takes "waist as the master", and the source of life is in the waist gap. Therefore, the practice of Tai Chi attaches great importance to the role of the kidney, takes the waist as the center to drive the coordinated movement of the limbs and the body, so that the movement of Qi is smooth. Tai Chi can make the waist get enough exercise to improve the circulation of Qi and blood in the waist, and make the kidney qi be continuously enriched, so as to treat kidney deficiency and improve immunity.

Long term practice of Tai Chi can help regulate nerves, smooth blood flow and promote metabolism, help improve immunity and enhance the resistance to the virus. Modern scientific research has found that when practicing Tai Chi, the central nervous system of the brain is in a state of excitation or inhibition. This relative effect can make people feel invigorated, refreshed and energetic after practicing Tai Chi. Moreover, long-term practice can make people open-minded, happy and wise. Through the effective cooperation of the boxing frame, idea and breathing, Tai
Chi can regulate the body's nerve function and immune system, effectively improve the cardiopulmonary function and enhance immunity.

\subsection{Effects of Tai Chi on cardiovascular diseases}

Chronic cardiovascular diseases account for a large proportion of the causes of abnormal death in middle-aged and elderly people. In the process of practicing Tai Chi, we must relax our body and mind without distractions. Makes the mind stable, thus makes the thinking quick, the language fluent. Tai Chi can promote the heart qi to run more smoothly, play its function of promoting the movement of blood, and then reduce and eliminate the congestion in the body. Modern medical research shows that Tai Chi can thicken cardiac muscle fibers, prevent cell fibrosis and degeneration, and thicken and strengthen the wall of the heart muscle, thus slow down the heart rate, strengthen the contractility of the heart muscle and improve the pumping function of the heart. It can effectively prevent chronic coronary heart disease, hyperlipidemia and other diseases. Therefore, through the exercise of Tai Chi, the heart function can be enhanced, the mind can be tranquilized, the diastolic period of the heart can be delayed, the myocardial can be fully rest, the myocardial contractility can be strengthened, the output can be increased, and the working ability of the heart can be improved. When practicing Tai $\mathrm{Chi}$, the movements are flexible, natural, soft and coordinated, which can increase the elasticity and stability of blood vessels, and effectively prevent hypertension and arteriosclerosis. Not only that, long-term practice of Tai Chi can also rhythmically contract and relax the muscles of the whole body, make the blood flow smooth, increase the venous return, thus accelerating the blood circulation, reducing the burden on the heart, and playing a role in the health care of the heart.

As for the capillaries, capillaries are the places of material exchange in microcirculation. Regular practice of Tai Chi can strengthen the microcirculation function, facilitate the material exchange inside and outside the capillaries, improve the utilization rate of oxygen in the tissues, reduce the accumulation of creatine, relieve fatigue. And it is beneficial to the recovery of diseases, especially for the prevention and treatment of chronic coronary heart disease, hyperlipidemia and arteriosclerosis. 


\section{THE EFFECT OF TAI CHI EXERCISE ON IMPROVING THE PROGNOSIS OF MIDDLE-AGED AND ELDERLY PATIENTS WITH CHRONIC DISEASES}

Tai Chi is a very gentle exercise, which not only has the effect of strengthening the body, but also plays a very good auxiliary role in the recovery of chronic diseases, and can gradually improve the physique of patients. At present, many sanatoriums and rehabilitation hospitals use Tai Chi for rehabilitation treatment. However, if we want to use Tai Chi to carry out targeted rehabilitation training for patients, it must be carried out under the correct care of doctors.

The calmness and tranquility required by Tai Chi is to eliminate distractions, so as to make people who are prone to irritability, depression and other abnormal emotions become calmer, reduce the adverse effects of negative emotions on patients. Calming the mind, relaxing the spirit, can reduce tension, improve adaptability, not only can eliminate tension and fatigue, but also can cultivate the body and mind. Relaxation of body and mind, can be conducive to the circulation of Qi and blood, promote blood circulation, thus play a role in restoring and improving health. Tai Chi can enable muscles and joints to complete a series of actions such as stretching and rotating at different angles, which can strengthen the supporting force of bones and the elasticity and toughness of muscles, improve the balance force, and increase strength and endurance. The overall movement law of Tai Chi is consistent with the law of modern human body, which is suitable for all kinds of people, especially those suffering from chronic diseases. It has been proved that practicing Tai Chi can improve mood, memory, character, sleep quality, movement stability, metabolic function and microcirculation. The practice of Tai Chi can not only get a healthy body but also a sound mind.

With the increase of age, middle-aged and elderly people will inevitably more or less suffer from different degrees of neurological decline and osteoporosis, and Tai Chi can effectively slow down these declines and improve neurological function. Studies have shown that after 6 months of Tai Chi practice, stroke patients' balance ability and postural control ability have been improved, which may be related to the adjustment of patients' proprioception by the practice. When practicing boxing, there are high requirements for the flexibility, flexibility and joint control strength of the limbs. Exercise can produce a good stimulation to the skeleton, help the elderly to improve muscle strength and increase flexibility.

\section{THE EFFECT OF TAI CHI ON THE DAILY HEALTH CARE OF MIDDLE-AGED PEOPLE}

Tai Chi, which is not affected by the external environment and low investment capital, has been widely liked by the middle-aged and old people. The daily health care effect of Tai Chi on the middle-aged and old people is also obvious. It can reduce the aging degeneration of the body or slow down its development process, so as to achieve the purpose of delaying aging and maintaining health.

Tai Chi exercise can significantly improve the cardiovascular, digestive, respiratory and nervous system functions. The most important thing is that it can improve the motor system functions of the middle-aged and elderly and the immune function to prevent diseases. After the human body enters the middle and old age stage, due to the obvious decrease of metabolism, the function of various organs will gradually decline. However, studies have shown that there is still a possibility to improve the structure and function of middle-aged and elderly people. One way is to take proper physical exercise to promote blood circulation and improve cell metabolism. One of the most effective exercise methods is Tai Chi. Regular practice of Tai Chi can strengthen the toughness of joints, improve the elasticity, flexibility and coordination of joints. It has a good effect on the prevention and treatment of muscle atrophy, ligament relaxation, reduction of synovial fluid secretion and joint stiffness caused by senile arthritis. Regular practice of Tai Chi can also make muscle fibers thicker, tough and powerful, It can also effectively improve the contractility, conductivity and reactivity of muscle fibers. It is reported that the decline in muscle working ability of middle-aged and elderly people who regularly practice Tai Chi is $10 \sim 20$ years later than that of non-practitioners. For middle-aged and elderly people, Tai Chi can exercise their physique, delay senility, extend working life and prolong life, so that they can give full play to their roles in society and family. More importantly, it can help middle-aged people gradually transition to the old age stage and better accept the colorful old life.

For normal middle-aged and old people, Tai Chi emphasizes relaxation, calmness and naturalness, and guiding the body through consciousness, so as to achieve "mind follows body" and "internal and external harmony", finally make people enter a relaxed situation, eliminate psychological fatigue and make people cheerful. Practicing Tai Chi also requires constant speed and slow movement, continuous movement in seeking static, static in moving, the combination of virtual and real combination, the combination of hardness and 
softness. Long time practicing Tai Chi can also make a person's character become steady, open-minded, calm, easygoing and optimistic. Through persistent practice of Tai Chi all year round, getting up early and coming home late in all weather, it can also cultivate the practitioner's perseverance and calm spirit, help to overcome laziness, disorientation, lack of concentration, weak will, negative personality and behavior habits, and reduce the possibility of various diseases.

\section{CONCLUSION}

To sum up, Tai Chi can strengthen vital qi and prevent diseases for middle-aged and elderly people without chronic diseases. For people who have already suffered from chronic diseases, it can relieve pain, improve the treatment effect and reduce the complications in the later stage of the disease. It has positive effects on the daily health care and treatment of middle-aged and elderly people suffering from chronic diseases. Under the current situation that everyone highly advocates traditional Chinese medicine for health preservation, Tai Chi deserves further recommendation.

\section{REFERENCES}

[1] Lai Sheguang. Tai Chi and health [N]. Yueyang Daily,2021-04-08(004).

[2] XIA Xiaobin. Discussion on the Health Value and Mechanism of Tai Chi $[\mathrm{J}]$. Chinese Martial Arts,2021(03):53-54.

[3] Zhu Buwen. Tai Chi and TCM health preservation [J]. Health Monthly,2020,41(10):906-908.

[4] WANG Jue. Discussion on the health preservation effect of Tai Chi from the perspective of traditional Chinese medicine [J]. Science and Technology Information,2020,18(16):208-209

[5] LI Zhiliang. Rambling on Tai Chi and health preservation [J]. Wudang,2020(05):16-20.

[6] Huang Ping. The guiding effect of Tai Chi situation culture connotation on modern health preservation [J]. Innovation Research of Snow and Ice Sports, 2020(04):94-95.

[7] Dong Fengwei, Wang Lihua. Theoretical significance and practical value of Tai Chi health preservation [J]. Journal of Nanjing Institute of Physical Education,2019,2(12):69-74.

[8] Ma Xinyu. Tai Chi and health care [J]. Jiangsu Health Care: Today Health Care, 2006, 000(004):53-53.

[9] YANG H. Discussion on the health preservation and fitness principle of Tai Chi [J]. Anhui Sports Science and Technology, 2004, 25(3):97-99.
[10] ZHAO F. On Tai Chi and modern health preservation [J]. Fighting: Wushu Science, 2007, 4(008):41-42.

[11] A. Pnueli, In transition from global to modular temporal reasoning about programs, in: K.R. Apt (Ed.), Logics and Models of Concurrent Systems, Springer, Berlin, Heidelberg, 1984, pp. 123-144. DOI: https://doi.org/10.1007/978-3-642-82453-1_5

[12] B. Meyer, Applying "Design by Contract", Computer 25(10) (1992) 40-51. DOI: https://doi.org/10.1109/2.161279

[13] S. Bensalem, M. Bogza, A. Legay, T.H. Nguyen, J. Sifakis, R. Yan, Incremental component-based construction and verification using invariants, in: Proceedings of the Conference on Formal Methods in Computer Aided Design (FMCAD), IEEE Press, Piscataway, NJ, 2010, pp. 257-256.

[14] H. Barringer, C.S. Pasareanu, D. Giannakopolou, Proof rules for automated compositional verification through learning, in Proc. of the 2nd International Workshop on Specification and Verification of Component Based Systems, 2003.

[15] M.G. Bobaru, C.S. Pasareanu, D. Giannakopoulou, Automated assume-guarantee reasoning by abstraction refinement, in: A. Gupta, S. Malik (Eds.), Proceedings of the Computer Aided Verification, Springer, Berlin, Heidelberg, 2008, pp. 135-148. DOI: https://doi.org/10.1007/978-3-540-70545-1_14 